\title{
Theory of Longitudinal Beam Halo in RF Linacs: II. Envelope-Particle Resonances
}

\author{
S. M. Lund \\ J. J. Barnard \\ This paper was prepared for submittal to the \\ 1997 Particle Accelerator Conference \\ Vancouver, B.C., Canada \\ May 12-16, 1997
}

May 9, 1997

This is a preprint of a paper intended for publication in a journal or proceedinge. Since changes may be made before publication, this preprint is made available with the underotanding that it will not be cited or reproduced without the permivion of the author. 


\section{DISCLAIMER}

This document was prepared as an account of work sponsored by an agency of the United States Government. Neither the United States Government nor the University of California nor any of their employees, makes any warranty, express or implied, or assumes any legal liability or responsibility for the accuracy, completeness, or usefulness of any information, apparatus, product, or process disclosed, or represents that its use would not infringe privately owned rights. Reference herein to any specific commercial product, process, or service by trade name, trademark, manufacturer, or otherwise, does not necessarily constitute or imply its endorsement, recommendation, or favoring by the United States Government or the University of California. The views and opinions of authors expressed herein do not necessarily state or reflect those of the United States Government or the University of California, and shall not be used for advertising or product endorsement purposes. 


\title{
THEORY OF LONGITUDINAL BEAM HALO IN RF LINACS: II. ENVELOPE-PARTICLE RESONANCES*
}

\author{
Steven M. Lund and John J. Barnard \\ Lawrence Livermore National Laboratory, L-440, Livermore, CA 94550
}

Abstract

Using the core/test-particle model described in a companion paper in these proceedings ${ }^{1}$ ("Theory of Longitudinal Halo in of Linacs: 1. Core/Test Particle Formulation," by J. J. Barnard and S. M. Lund), we analyze longitudinal beam halo produced by resonant self-field interactions in intense, ion-beam of linacs. It is shown that particles moving in the presence of the space-charge forces of an oscillating, mismatched ellipsoidal beam bunch can be resonantly driven to large longitudinal amplitude. This resonantly produced halo is first analyzed in a limit where it is most simply understood, with particles moving purely longitudinally and with linear $r f$ focusing. Then modifications of the resonance induced by nonlinear $\mathrm{rf}$ and transverse-longitudinal coupling are explored.

\section{INTRODUCTION}

Resonant interactions between particle orbits and oscillating space-charge forces associated with a mismatched beam core are known to be a significant cause of transverse $(\perp)$ beam halo ${ }^{I 2}-I 4,2-4$. Recently, simulation work using idealized core/particle models has suggested that analogous, resonantly produced halo may occur longitudinally and be an issue of concern in intense-beam applications ${ }^{2}$. Here, we examine resonantly produced longitudinal (II) halo uaing a more detailed core/particle model developed in an accompanying paper (Ref. 1, hereafter referred to as Paper I). In Paper I it was shown that a low-frequency mode (LFM) of a mismatched ellipsoidal beam bunch with uniform space-charge can drive a low-order longitudinal resonance (LR) with analogous properties to that of $\perp$ beam halo. To understand the essential physics that determines the structure of the resulting \| halo, the LR is numerically and analytically analyzed under increasingly realistic conditions in Secs. II-IV.

The notation employed in this paper is developed in Paper I. Specific references to citations and equations from Paper I will be denoted by the prefix I. All model results are illustrated using the parameters of the $100 \mathrm{MeV}$ point of an intense proton-beam linac presented in the Table of Paper I. This linac corresponds to the normal conducting design originally considered for the Accelerator Production of Tritium (APT) project.

\section{LINEAR RF FOCUSING}

To simply elucidate the essential features of the LR, a linear rf approximation is taken [see Eq. (I-5)] where the $\|$ focusing force is linear about displace-

*Work performed under the auspices of the U.S. D.O.E. by LLNL under contract W-7405-ENG-48
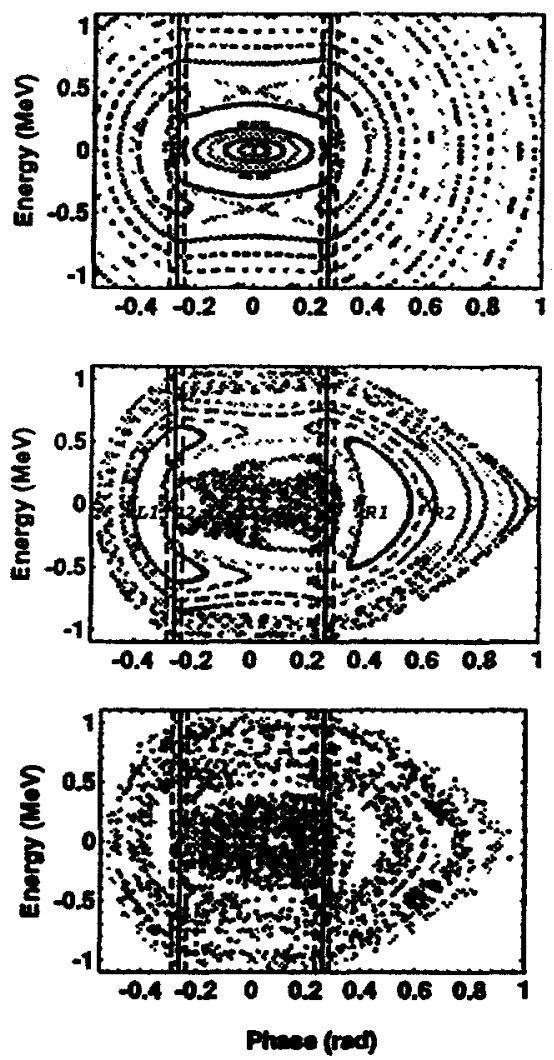

Fig. 1. Poincaré plots of energy $(\Delta \mathcal{E})$ and phase $(\Delta \phi)$ phase-space for $\delta r_{s} / r_{s 0}=0.1, I=200 \mathrm{~mA}$, and: $a$. (top) Linear if and $x_{\perp}=0$. b. (middle) Nonlinear if and $x_{\perp}=0$. c. (bottom) Nonlinear if and $x_{\perp} \neq 0$.

ments from the synchronous particle, envelope motion is assumed to be in a pure linear LFM (as opposed to a general linear mode superposition of a LFM and HFM, or a finite amplitude solution to the full nonlinear envelope equations), and only on-axis particles (i.e., $x_{\perp}=0$ ) are considered. To visualize the structure of the $L R$ in $\Delta \phi-\Delta \mathcal{E}$ phase-space, particles are initialized with $\Delta \mathcal{E}=0$ and $\Delta \phi$ uniformly distributed over half the phase-width of the if bucket and Poincaré plot images of the particle phase-space are formed by numerically integrating Eqs. (I-4) with respect to $s$ and superimposing snapshots of the I| phase-space accumulated at $s$ where the excursion of the $\|$ envelope oscillation is minimum (at $s$-increments of $2 \pi / k_{L}$ ). A typical Poincaré plot produced by accumulating long enough to clearly visualize the LR is presented in Fig. 1(a) for 20 particles and an envelope mode amplitude of $\delta r_{z} / r_{x 0}=0.1$. The solid vertical lines indicate the phase width of the equilibrium beam radius $r_{x 0}$ and the dashed vertical lines indicate the phase extent of the envelope oscillations. Particles deep within and far outside the core of the beam undergo simple 
depressed and undepressed synchrotron oscillations, respectively. At intermediate amplitudes there is a strongly expressed resonant structure in which particles undergo approximately two oscillation periods over a LFM envelope period, thereby identifying the LR suggested in Paper I. Higher-order resonances with smaller amplitudes also appear. Such resonances become more significant and interact with the LR with increasing space-charge forces (i.e., increasing $I$ ).

In analogy to Gluckstern's analysis of transverse beam halo $^{I-3}$, the LR can be analyzed analytically if, in addition to the assumptions above, a limit of a spherical bunch in the beam frame (i.e., $\gamma_{t} r_{z 0}=r_{\perp 0}$ ). is taken. In this case, the space-charge force exterior to the bunch [see Eq. (I-1)] has a simple inverse-square fall-off $\sim \Delta z /|\Delta z|^{3}$ exterior to the bunch, and the equations of motion (I-4) can be expressed in a phaseamplitude form and with all non-resonant phases averaged over to derive a phase-averaged constant of the motion $^{I-6}$. This constant can be interpreted as a resonant particle Hamiltonian given by

$$
\begin{gathered}
H=-\left(2 k_{,}-k_{x}\right) w+\frac{k_{c c}^{2}}{\pi k_{*}}\left\{(w-6)\left[\pi-2 \sin ^{-1}\left(\frac{1}{\sqrt{w}}\right)\right]\right. \\
\left.+2 \sqrt{w-1}+\frac{4}{\sqrt{w}} \ln \left(\frac{\sqrt{w}+\sqrt{w-1}}{\sqrt{w}-\sqrt{w-1}}\right)\right\} \Theta(w-1)-\frac{3 k_{c o}^{2}}{2 k_{*}}\left(\frac{\delta r_{x}}{r_{x 0}}\right)\left\{\frac{1}{2}\right. \\
\left.+\frac{1}{\pi}\left[\sin ^{-1}\left(\frac{1}{\sqrt{w}}\right)-\frac{\pi}{2}-\frac{\sqrt{w-1}(w-2)}{w^{2}}\right] \Theta(w-1)\right\} w \cos \Psi
\end{gathered}
$$

where $k_{s c}=\sqrt{K_{3 D} / 3 \gamma_{s}^{2} r_{x 0}^{3}}$ is a space-charge wavenumber, $\Theta(w-1)$ is a $\theta$-function with $\Theta(w-1)=1$ for $w>1$ and $\theta(w-1)=0$ for $w<1$, and $(w, \Psi)$ are coordinates related to the $(\Delta \phi, \Delta \mathcal{E})$ coordinates as

$$
\begin{aligned}
w & =\left(\frac{\Delta \phi}{\Delta \phi_{b}}\right)^{2}+\left(\frac{\Delta \varepsilon}{\Delta \varepsilon_{b}}\right)^{2}, \\
w \cos \Psi & =\left(\frac{\Delta \varepsilon^{2}}{\Delta \varepsilon_{b}^{2}}-\frac{\Delta \phi^{2}}{\Delta \phi_{b}^{2}}\right) \cos k_{L} s+\frac{2 \Delta \phi \varepsilon \varepsilon}{\Delta \phi_{b} \Delta \varepsilon_{b}} \sin k_{L} s .
\end{aligned}
$$

Here, $\Delta \phi_{b} \equiv 2 \pi r_{z 0} / \beta_{s} \lambda$ and $\Delta \mathcal{E}_{b} \equiv m c^{2} \gamma_{s}^{3} \beta_{s}^{2} k_{s} r_{s 0}$ are the equilibrium bunch widths in phase and energy. The resulting LR phase-space generated by the $H=$ const. surfaces of this spherical bunch conservation constraint is plotted in Fig. 2 for $1=50 \mathrm{~mA}$ (other parameters fixed) and with the characteristic frequencies $k_{z}, k_{z c}$ and $k_{L}$, equilibrium radius $r_{z 0}$, and mode amplitude $\delta r_{z} / r_{z 0}$ taken from the ellipsoidal bunch problem. The corresponding numerically calculated Poincaré plot of the ellipsoidal bunch is shown in overlay. The rough agreement between these solutions can be understood in terms of an insensitivity of the resonant structure with respect to the precise nature of the I| space-charge force. For example, if the inversesquare fall-oft in the exterior space-charge force law is replaced by one with a more rapid falloff, similar conservation constraints can be derived and little change is observed in the resonance structure. However, this agreement appears to break down at higher bunch currents $I$, suggesting a limit to this argument that has yet to be quantified in detail.

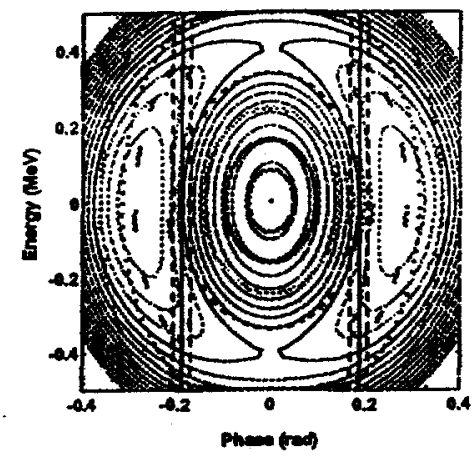

Fig. 2. Overlay of theoretical (spherical bunch) and simulated (elliptical bunch) LR energy $(\Delta \mathcal{E})$ and phase $(\Delta \phi)$ phase-space for linear $\mathrm{rf}, \mathrm{x}_{\perp}=0, I=50 \mathrm{~mA}$.

\section{NON-LINEAR RF FOCUSING}

The linear if approximation fails as distance from the synchronous particle is increased. Moreover, even the equilibrium bunch can occupy a significant fraction of the if wavelength. In this section, modifications of the LR induced by the sinusoidal nature of the applied of focusing are analyzed numerically. For simplicity, all assumptions outside of the linear if focusing limit of the ellipsoidal beam analysis in Sec. II are maintained (i.e., on-axis particles and a linear LFM envelope mode). The phase space obtained is presented in Fig. 1(b) for the same parameters as Fig. 1(a). Note that the structure now has a left-right asymmetry associated with the anharmonic nature of the if focusing and is well confined within the separatrix of particle loss for the if bucket. At larger mismatch amplitudes $\delta r_{x} / r_{30}$ the structure becomes broader and moves closer to bucket seperatrix, but for $\left|\delta r_{z}\right| / r_{s 0}<0.5$ does not lead to particle loss from the bucket. This result is illustrated in Fig. 3, where the phase-variation (in $\Delta \phi$ ) of labeled structure features is plotted as a function of mismatch amplitude $\left|\delta r_{x}\right| / r_{z 0}$. Note that the width of the resonant structure (as measured by the phase widths of $X_{L 1}$ to $X_{L 2}$ and $X_{R 1}$ to $X_{R 2}$ ) increases with amplitude and approaches the beam core, while the structure O-point varies little with amplitude. At large amplitudes, the close proximity of the LR structure to the core $\left( \pm \Delta \phi_{\text {eq }}\right.$ $=$ phase edges of core) suggests that it will be easily populated, potentially degrading beam quality. However, as a consequence of the nonlinear field structure, halo extent appears to remain confined within the extent of the rf bucket (indicated with $S_{ \pm}$markers).

When the bunch current $I$ is varied with nonlinear rf focusing, an interesting bifurcation of the LR is observed. Namely, at low- $(I<22.1 \mathrm{~mA})$ and highcurrents $(I>284 \mathrm{~mA})$, the low-order LR disappears and a smaller-amplitude, higher-order resonance dominates. This situation can be understood with reference to Fig. 4. Here, the maximum particle frequency $k_{M}$, which occurs exterior to the beam core, is numerically calculated as a function of $I$ (with other parameters fixed). Note that for low and high currents, half the 
LFM frequency $\left(k_{L} / 2\right)$ falls above the maximum frequency $k_{M}$, thereby precluding the LR (see arguments in Paper I). Note that higher-order resonances are still possible for $I>283 \mathrm{~mA}$ as demonstrated by the $k_{L} / 3$ curve falling between $k_{M}$ and the depressed core frequency $k_{s}$. Consistent with Fig. 4 , no resonances are observed for $I<22.1 \mathrm{~mA}$.

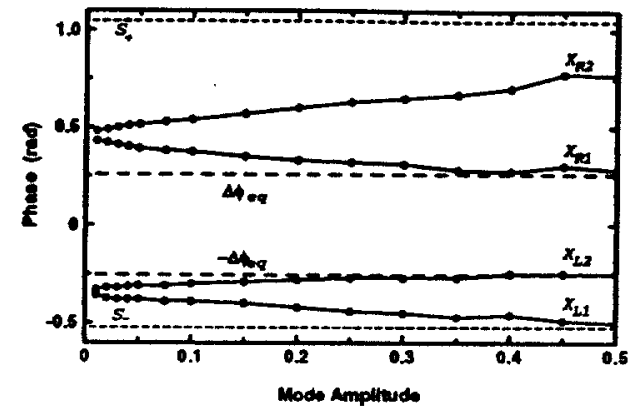

Fig. 3. Phase $(\Delta \phi)$ measures of LR features versus mode amplitude $\left(\left|\delta r_{z}\right| / r_{z 0}\right)$ for nonlinear $\mathrm{rf}, \mathrm{x}_{\perp}=0$, $I=200 \mathrm{~mA}$.

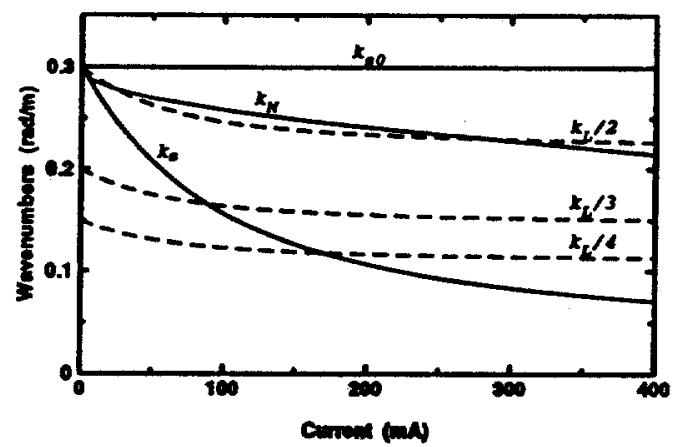

Fig. 4. Maximum particle $\left(k_{M}\right)$, depressed and undepressed synchrotron $\left(k_{2}\right.$ and $\left.k_{30}\right)$, and subharmonic LFM envelope mode $\left(k_{L} / 2, k_{L} / 3\right.$, and $\left.k_{L} / 4\right)$ frequencies versus bunch current $I$ with nonlinear $\mathrm{rf}$.

\section{EFFECTS OF $\perp / \|$ COUPLING}

Since the accelerator beams have finite $\perp$ emittance, particles will in general have finite $\perp$ betatron oscillations $\left(x_{\perp} \neq 0\right)$ and, outside the bunch, this motion will introduce $\perp / \|$ coupling in the particle equations of motion. This coupling is included in the core/ particle model derived in Paper I. In this section, we analyze the effects of this $\perp / \|$ coupling on the LR. In Fig. 1(c), we show Poincare phase space plots for the same parameters as Fig. 1(b), with the exception that all particles are initialized at $x=0.6 r_{\perp 0}$ instead of $\mathbf{x}_{\perp}=0$. Notice that the resonance structure persists, although it is somewhat smeared.

It has been noted ${ }^{3}$ that under some circumstances || particle oscillation amplitudes can be converted into larger $\perp$ amplitudes. We confirm the qualitative observation that conversion occurs, but fail to confirm the magnitude of the effect. We have simulated three particles with initial values of $x / r_{10}=0.4,0.94,2.5$, and one particle with an initial value that was at 0.80 of the maximum || phase to remain within the stable bucket, (corresponding to $x / r_{10}=3.0$ ). These val- ues were similar to the values chosen in the example in Ref. 3, although the overall accelerator and beam parameters were different than in our simulations. Additionally, as in Ref. 3, we initialized the modes such that the initial perturbation satisfied $\delta r_{z} / r_{x 0}=0.1$ and $\delta r_{\perp} / r_{\perp 0}=0.1$, and numerically integrated the equations of motion (I-4) for the four particles under these assumptions. Fig. 5 illustrates the transverse $\left(x-x^{\prime}\right)$ phase-space obtained (using linear if focusing) while strobing at the frequency of the HFM. The two particles with the large initial || amplitudes increased their $\perp$ radius by a factor of approximately 1.8 , reaching amplitudes which are slightly exterior to the beam radially. This is in contrast to factors of up to 30 seen in Ref. 3. For these parameters, a factor of 1.8 is consistent with particle motion bounded by the equipotential corresponding to the total energy of a particle in the potential well of an unmismatched beam ${ }^{I-6}$. The main effect of the coupling appears to be allow the particle to access a larger fraction of the available phase-space bounded by the total potential in the absence of mismatch.

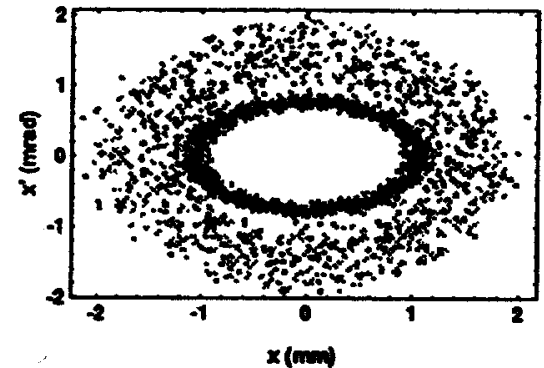

Figure 5. Poincaré plot of transverse phase-space of four particles with different initial $\Delta z$, but with identical initial $x=0.6 r_{\perp 0}=1.1 \mathrm{~mm}$.

\section{CONCLUSIONS}

The core/particle model developed in Ref. 1 has been employed to explore \| beam halo due to resonant interactions with envelope oscillations. Characteristic frequencies of particle and envelope oscillations play a critical role in determining the extent and structure of the halo. Model predictions of halo extent are shown to persist under increasingly realistic conditions.

\section{ACKNOWLEDGMENTS}

The authors wish to again thank $R$. Ryne, $T$. Wangler, and R. Gluckstern and in addition note helpful discussions with D. Bruhwiler ( $\perp / \|$ coupling) and C. Chen, A. Friedman, and I. Hofmann.

\section{REFERENCES}

[1] J.J. Barnard and S.M. Lund, "Theory of Longitudinal Beam Halo in RF Linacs: I. Core/Test-Particle Formulation," these proceedings.

[2] T.P. Wangler, R.W. Garnett, E.R. Gray, R.D. Ryne, and T.S. Wang, Proc. of the XVIII Int. Linear Accel. Conf., Geneva, 1996, p. 372.

[3] D.L. Bruhwiler, loc. cit. 2.

[4] M. Pabst and K. Bongardt, loc. cit. 2. 


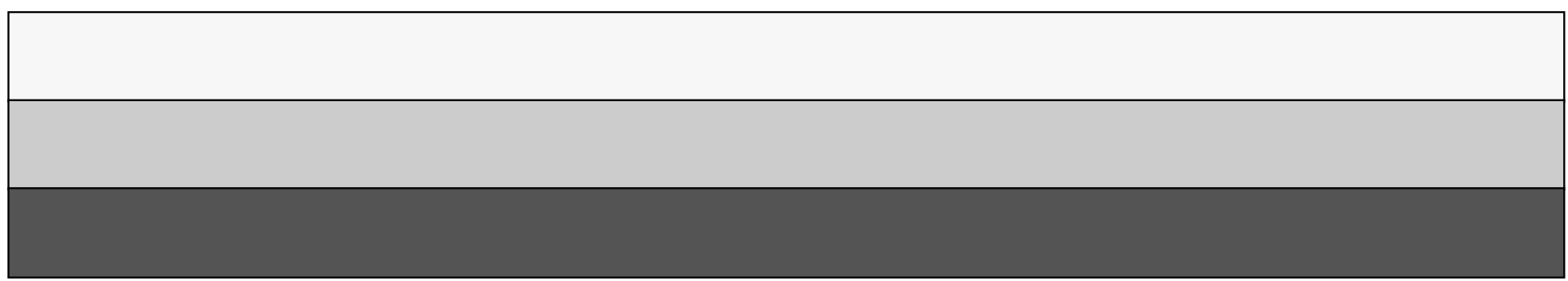

